\title{
Test Facilities in Support of High Power Electric Propulsion Systems
}

\author{
Melissa Van Dyke, Mike Houts, Thomas Godfroy, Ricky Dickens, James J. \\ Martin, Patrick Salvail, and Robert Carter
}

Marshall Space Flight Center, National Aeronautics and Space Administration, Huntsville, Alabama, 35812

(256) 544-5720, Melissa.Vandyke@msfc.nasa.gov

\begin{abstract}
Successful development of space fission systems requires an extensive program of affordable and realistic testing. In addition to tests related to design/development of the fission system, realistic testing of the actual flight unit must also be performed. If the system is designed to operate within established radiation damage and fuel burn up limits while simultaneously being designed to allow close simulation of heat from fission using resistance heaters, high confidence in fission system performance and lifetime can be attained through non-nuclear testing. Through demonstration of systems concepts (designed by DOE National Laboratories) in relevant environments, this philosophy has been demonstrated through hardware testing in the High Power Propulsion Thermal Simulator (HPPTS). The HPPTS is designed to enable very realistic non-nuclear testing of space fission systems. Ongoing research at the HPPTS is geared towards facilitating research, development, system integration, and system utilization via cooperative efforts with DOE labs, industry, universities, and other NASA centers. Through hardware based design and testing, the HPPTS investigates High Power Electric Propulsion (HPEP) component, subsystem, and integrated system design and performance.
\end{abstract}

\section{INTRODUCTION AND BACKGROUND}

To allow early utilization, system designs must be relatively simple, easy to fabricate, and easy to test using nonnuclear heaters to closely mimic heat from fission. This combination of attributes will allow pre-prototypic systems to be designed, fabricated, and tested quickly and affordably. The ability to build and test units is key to the success of a nuclear program, especially if an early flight is desired. Designing systems to be highly testable using nonnuclear heaters to closely mimic heat from fission may help avoid the need for a series of expensive and timeconsuming full power ground nuclear tests. Required data would be obtained from a combination of full-power nonnuclear heated tests, zero-power criticals, and in-pile testing of components or modules. Even if it is still determined that a full power ground nuclear test is needed, the ability to perform very realistic non-nuclear testing prior to that test would increase the success probability of the full power ground nuclear test.

The reactor thermal power level proposed for most early demonstration missions is $<400 \mathrm{kWt}$. At these power levels, systems can be designed such that most potential issues are thermal or stress related, with nuclear effects being secondary. More specifically, the reactors can be designed such that fuel burnup is within demonstrated capability, and designed such that the fast neutron fluence seen by reactor materials and components is well below the level where significant radiation damage would occur. If these design criteria are met, realistic and valuable testing can be performed using non-nuclear heaters to closely mimic heat from fission.

The focus of the hardware based effort within NASA Marshall Space Flight Center's Propulsion Research Center is on enabling early utilization of space fission systems. The result of this focus has been the development of fabrication and test capabilities necessary for rapidly building and testing potential near-term space fission systems. This capability includes advanced thermal simulators, an extremely versatile test chamber, high purity alkali metal handling capability, and advanced manufacturing (e.g. e-beam welding, high temperature braze).

\section{High Power Propulsion Thermal Simulator (HPPTS)}


The HPPTS describes the facilities at MSFC that are used to demonstrate very realistic non-nuclear testing of space fission systems. This includes, but is not limited to, a $9 \mathrm{ft}$ diameter and a $2 \mathrm{ft}$ diamater vacuum chamber. Ongoing research at the HPPTS is geared towards facilitating research, development, system integration, and system utilization via cooperative efforts with DOE labs, industry, universities, and other NASA centers.

Through hardware based design and testing, the HPPTS investigates High Power Electric Propulsion (HPEP) component, subsystem, and integrated system design and performance. Previous non-nuclear tests in the HPPTS have proven to be a highly effective method (from both cost and performance standpoint) to identify and resolve HPEP integration issues. For example, in designing the test plenums for a heat pipe reactor heat exchanger concept and for the Testable Direct Gas Cooled Reactor flow channels concept, several stress and heat transfer issues were identified resulting in a redesign of the proposed heat exchanger for the heat pipe reactor and of the gas entrance plenum for the gas cooled reactor. Because both of the involved DOE laboratories (Los Alamos and Sandia) are testing specific parts of their designs, DOE modified their reactor/heat exchanger designs used in the current DOE systems trade studies.

All of the current HPPTS hardware work supports a systems integration testbed that allows prototypic testing of the systems under investigation. Each year, the testbed provides prototypic products that feed into the current systems integration trade studies/design. The testbed is built such that when new components become available, they can readily be incorporated into the system without having to build new facilities and train people. By working in conjunction with the DOE labs, both sets of expertise (e.g. LANL/Sandia for nuclear design and MSFC for testing) can be utilized. As of the end of FY02, the HPPTS will have demonstrated the capability to test heat pipe cooled reactors and direct gas cooled reactors, two leading options for use on an early flight. Preliminary work will also be completed on designing and testing a stainless steel based pumped $\mathrm{NaK}$ system, which is a third potential option for an early flight.

\section{Full Scale Testing - Laboratory C}

Full scale testing is performed in the $9 \mathrm{ft}$ diameter (18 ft barrel length with elliptical domes) vacuum facility (Figure 1). This facility, driven by 4 diffusion pumps $(32,000 \mathrm{Vs}$ each) and 3 roughing pumps $(34,000 \mathrm{Vs}$ each), is capable of vacuum levels of $10^{-6}$ torr or better. All diffusion pumps and roughing lines are equipped with chill water baffles to prevent back streaming of pumping oil. The vacuum chamber is equipped with a large "vacuum relief" sliding blank flange positioned on top of the chamber to protect against inadvertent overpressure. The chamber also contains blank flanges for hardware tests requiring feed-throughs, regulators, isolation valves and connection hardware. The chamber is equipped with an external video camera (on a vacuum rated view port) and internally with electrical lighting.

Power is provided to the test article via an automated 32-zone power and control system. The zones allow very accurate matching of the predicted core radial nuclear power profile. The zones operate at low voltage (150 V maximum) to allow testing to occur in vacuum or with any desired gas mixture and pressure. While the concept of resistance heating is not new, MSFC is currently the only place in the United States where realistic non-nuclear tests of potential space reactor cores and integrated systems can be performed. MSFC has demonstrated the ability to realistically simulate a heated core in both a vacuum and a $\mathrm{CO}_{2}$ environment through demonstration of a full-scale, unfueled reactor core coupled to a power conversion subsystem. Testing of cores and core mockups containing from 48 to 183 fuel pins has been completed. Testing in a helium atmosphere has also been performed in order to benchmark reactor heat transfer predictions.

To simulate the heat from fission, an electrical power system is required to feed sufficient power to allow allowing for test article operation at full power with sufficient margin to compensate for losses. This system uses a switchboard to feed a power distribution network and finally a large bank of power supplies (Figure 2). Circuit breaker protection is provided at each level of this network and all power supplies are equipped with protection for both over current and over temperature conditions. The DC power supplies are controlled by independent programmable logic controllers with preprogrammed red lines and logic to compensate for loss of communication with the computer based Human Machine Interface software (that provides the remote operation). This system is strategically positioned behind the vacuum chamber and uses overhead wire ways to route power to the vacuum 
chamber (maximizing workability while minimizing the safety hazard). Additionally, separate facility power is provided to operate vacuum pumps, water pump, data/control systems and other test article items. Although the control system is capable of delivering $480 \mathrm{~kW}$, more power is available and can be tapped from the $1.5 \mathrm{MW}$ switchboard by adding extra power supplies to the system.

A closed loop gas conditioning system exists that can be utilized for any test article. Although the system is designed for a $\mathrm{He} / \mathrm{Ar} \mathrm{mix}$, it can accommodate other gases as well. Currently the system is designed for $.09 \mathrm{to} 0.2 \mathrm{~kg} / \mathrm{sec}$ flowrate, 600 to $900 \mathrm{~K}$ inlet temperature (to test article), 850 to $975 \mathrm{~K}$ out (from test article), and a gas pressure between 1.0 to $2.5 \mathrm{Mpa}$.

For pre-experiment assembly, approx. $200 \mathrm{sq} \mathrm{ft}$ of environmentally controlled floor space in the front of the vacuum chamber is available to setup data acquisition, control, power, etc.. There is approx. $200 \mathrm{sq} \mathrm{ft}$ of space on the door end of the vacuum chamber to stage hardware. There is approx $50 \mathrm{sq} \mathrm{ft}$ of floor space opposite the door end of the vacuum chamber to accommodate thegas-handling system. Team members have a 24-hour a day access to the facility.

The HPPTS facility (Laboratory C) also has a dedicated machine shop with the following machines: lathes (2), mill, table saw, cut-off saw, portable welder, welding booth, grinder, tube bender, and tube flaring machine

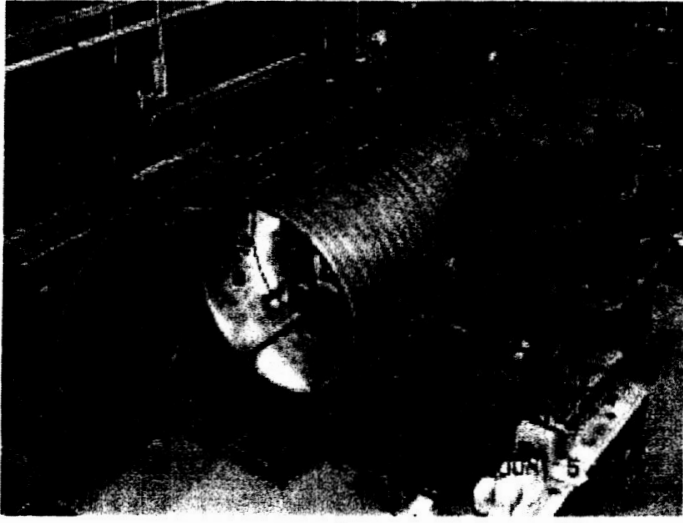

FIGURE 1. $9 \mathrm{ft}$ Vacuum Chamber .

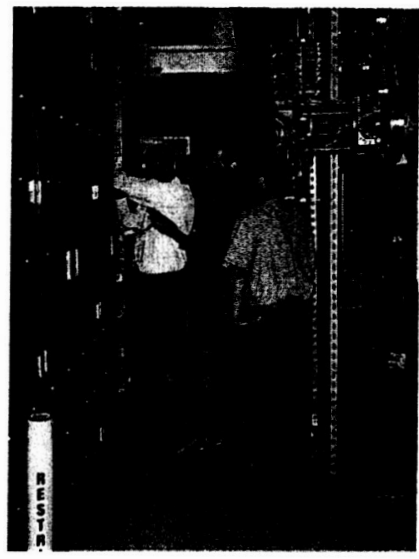

FIGURE 2. Power System in Laboratory C.

\section{Advanced Thermal Simulators}

Experimental verification of heat transfer and stress issues is best performed if non-nuclear thermal simulators can be used to very closely mimic heat from fission. To accomplish this, the thermal simulators must be able to fit within the nuclear fuel pin cladding, must be able to match the predicted axial nuclear power profile of the fuel pin, and must be able to match the effective radial conductivity of the fuel pin.

To meet these criteria, two types of thermal simulators are being developed at NASA MSFC. One is an alumina sleeved graphite heater with a minimum outer diameter of 0.365 ". The baseline heater is designed to operate at 1300 $\mathrm{W}$, with a peak temperature capability $>1700 \mathrm{~K}$. The design allows for axial power profiling and radial conductivity matching. Heater testing has included rapid cycling, 8 hour runs, and 24 hour runs. Cumulative test time for individual heaters is approaching 500 hours.

The other type of thermal simulator is an alumina (or sapphire) sleeved spiral wound refractory alloy wire design. Potential advantages of this approach include smaller minimum outside diameter ( $<0.30$ ") and ease of attaining smooth axial power profiling. This design also allows for axial power profiling and radial conductivity matching. Figure 3 is a picture of this thermal simulator test. Because of the uniqueness of the thermal simulators, MSFC has patents pending on both the heating elements and the power lead assemblies. 


\section{Laboratory B: Modular Testing and Heater Development}

The primary focus of testing in this laboratory is modular level problem solving, small-scale concept demonstration, and heater research. The first non-nuclear test at MSFC was conducted in this chamber. The Module Unfueled Thermal hydraulic Test (MUTT) was a single refractory metal module with lithium heatpipe which demonstrated over $1500 \mathrm{deg} \mathrm{K}$ in excess of 100 hours and over 10 restarts.

This vacuum chamber is a 24 " diameter, 6 foot long, cylindrical water jacket cooled stainless steel vacuum chamber capable of operating at pressures below $1.0 \times 10^{-7}$ Torr. The vacuum chamber is able to rotate vertically about its center axis and is mounted on a mobile support frame that facilitates quick and easy movement of the entire chamber. There are 16, 2-3/4" Conflat Flange ports, nine 6" Conflat, and one 8" Conflat ports located symmetrically about the chamber. These ports are used as viewports for visual inspection of the article or for optical data collection as well as feedthrough ports for data, gas, and power. The vacuum system is connected to the largest port. Due to the high temperature / high power nature of most simulated nuclear testing, the chamber is equipped with a water jacket to remove heat from the chamber walls produced during testing. Flow rates to both the chamber and the pumps are measured with flow meters and monitored by a control system.

Module testing of a portion of a full-scale systems tested in the $9 \mathrm{ft}$ vacuum chamber are performed here. For example, when the SAFE 30 (heat pipe system consisting of 12 modules) heat transfer optimization study was being worked, 1 module was tested in the small vacuum chamber. When the power system for the $9 \mathrm{ft}$ vacuum chamber was being designed. One of the 32 control zones were built, tested, and verified in this small vacuum chamber before acquiring the entire system.

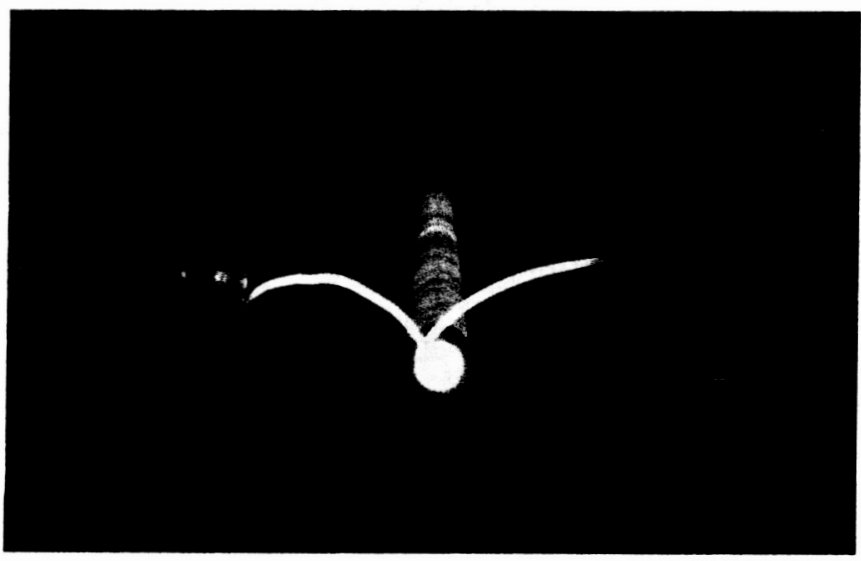

FIGURE 3. Thermal Simulator Development at NASA MSFC.

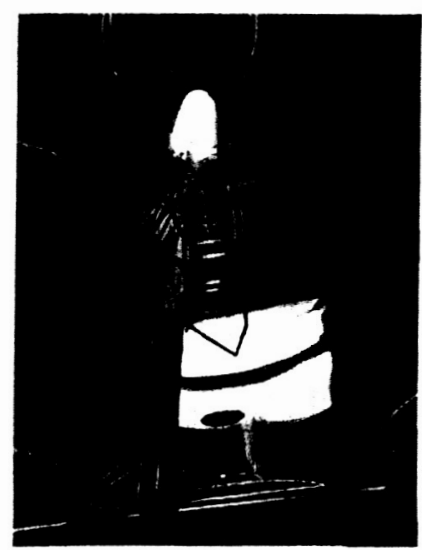

FIGURE 4. Module Testing in Laboratory B.

\section{High Purity Alkali Metal Handling and Fill}

High purity alkali metals have several potential applications. For early systems, potassium or sodium heat pipes could be used for primary heat transport. Early systems could also utilize a stainless steel based pumped NaK system for primary heat transport, with the working fluid being $78 \%$ potassium and $22 \%$ sodium. Early (and futuristic) fission reactor cores may also utilize alkali metal heat pipes for decay heat removal, thermal management, and providing power to backup power converters. Fabrication and test of these systems will require high purity alkali metal handling and fill capability. More futuristic systems may require the use of high purity lithium. These include systems with heat pipes operating at $>1400 \mathrm{~K}$, or advanced refractory metal pumped lithium loops. Advanced systems using potassium-Rankine power conversion will also require a source of high purity potassium.

MSFC currently has the capability to handle and fill high purity sodium. Potassium handle and fill capability can easily become available in FY03. Lithium capability can be added on an as-needed basis. MSFC capability in this 
area could be particularly useful if an early-flight scenario was desired, in that because the capability already exists, it should be possible to shorten fabrication and test schedules.

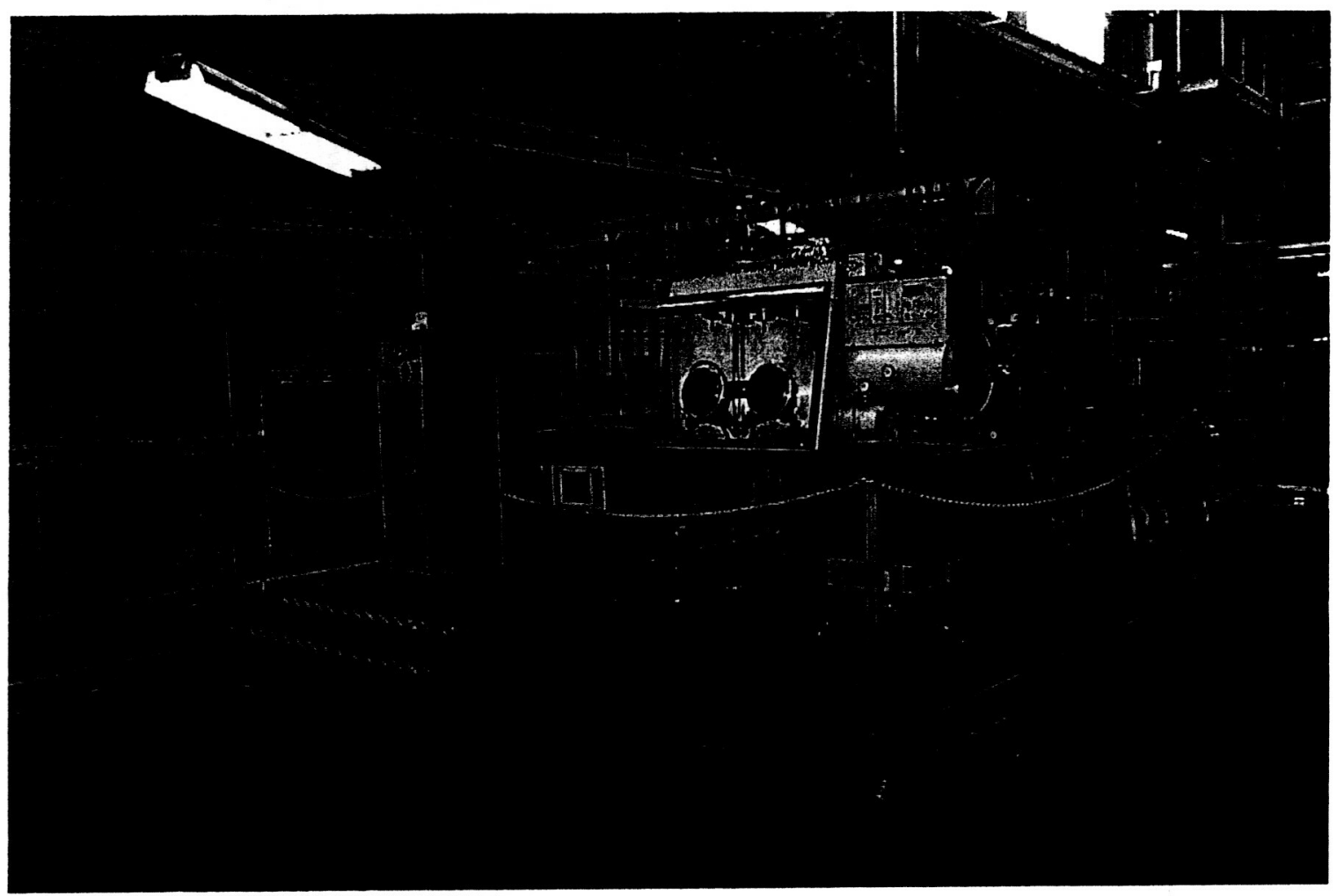

FIGURE 5. High Purity Alkali Metal Fill and Handling.

\section{MSFC Manufacturing Capability}

In addition to dedicated test facilities used for the HPPTS, other facilities at MSFC in the manufacturing area are also utilized to support this effort. Specific capabilities utilized to date include, but are not limited to, the following.

- Thermal Mechanical Processing Facilities - including Vacuum induction melt Furnace, Roll Mill, Hammer Forge, Various Heat-Treat and Brazing Furnaces, Hot Isostatic Press (HIP).

- Mechanical Testing Facilities - Various Mechanical Testing Equipment including Tensile Testers, Hardness Testers, etc.

- Microscopy/Failure Analysis Facilities - instruments including Scanning Transmission Electron Microscope (STEM), Field Emission Scanning Electron Microscope (FESEM), Electron Spectroscopy for Chemical Analysis (ESCA), and other optical instruments.

- Plating Facilities - electrodeless plating.

- Welding/Machining Facilities - Welding, Machining, and Manufacturing.

- Vacuum Plasma Spray Facilities

- Rapid Prototyping

- Manufacturing Facilities 


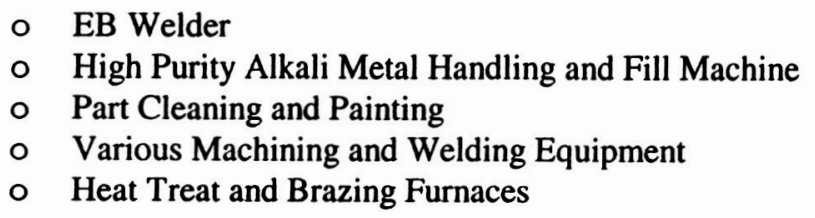

MSFC manufacturing facilities have to date been used in support of the $30 \mathrm{kWt}$ Safe Affordable Fission Engine (SAFE-30) project, the SAFE-100 project, heat exchanger design and fabrication (needed for heat pipe cooled or pumped liquid metal cooled systems), the Testable Direct Gas Cooled Reactor project, and other activities.

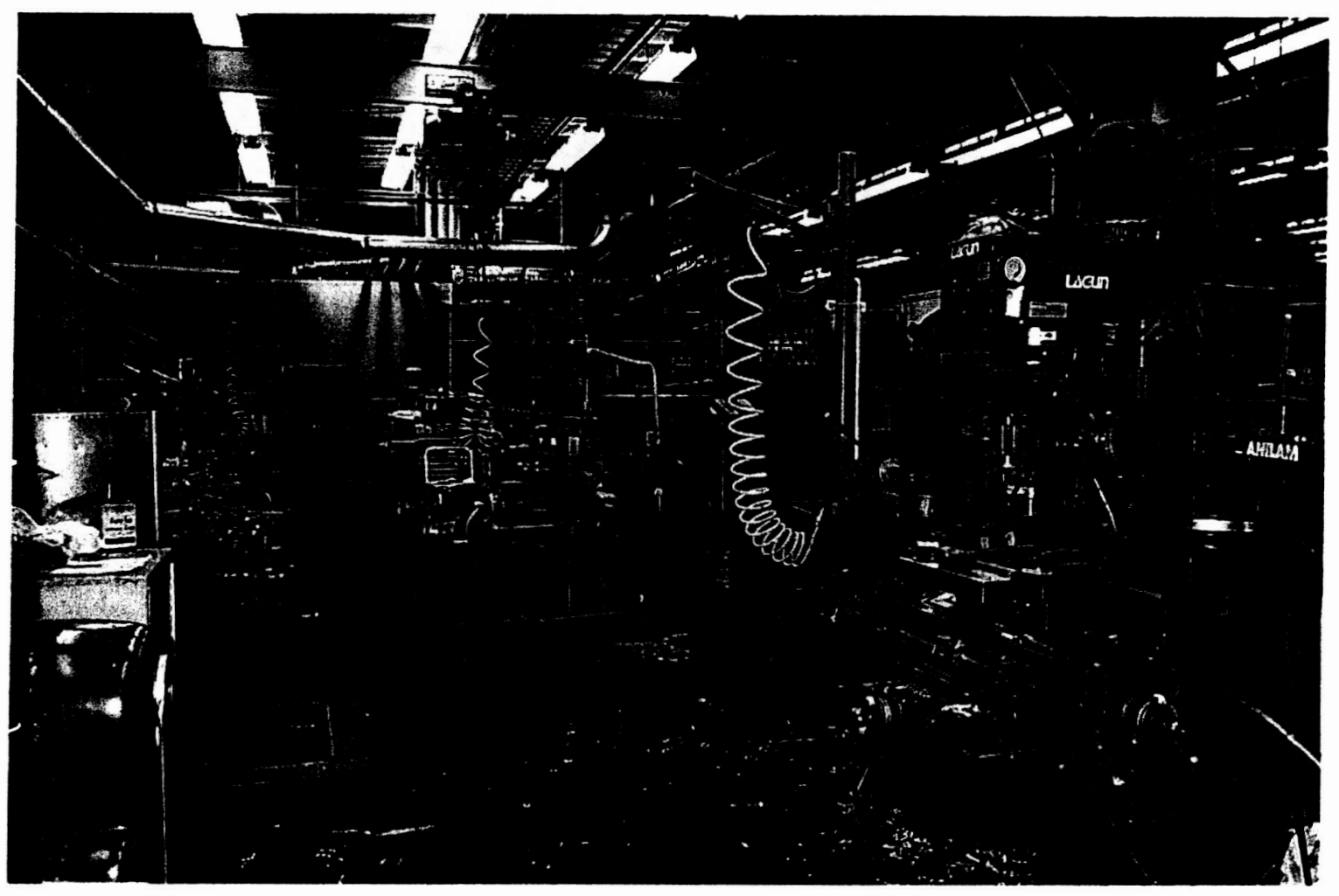

FIGURE 6. Portion of an MSFC Machine Shop.

\section{SUMMARY}

The focus of non-nuclear research and development tresting within NASA Marshall Space Flight Center's Propulsion Research Center is on enabling early utilization of space fission systems. The result of this focus has been the development of fabrication and test capabilities necessary for rapidly testing potential near-term space fission systems. This capability includes advanced thermal simulators, extremely versatile test chambers, high purity alkali metal handling capability, and advanced manufacturing (e.g. e-beam welding, high temperature braze).

\section{REFERENCES}

Van Dyke, M., Houts, M., Pedersen, K., Godfroy, T., Dickens, R., Poston, D., Reid, B., Salvail, P.,Ring, P., "Phase 1 Space Fission Propulsion System Testing and Development Progress," in proceedings of Space Technology and Applications International Forum edited by M. El-Genk, AIP 552, AIP, New York, 2001, pp 837-842. 